\title{
Retraction Note to: Robust Model Selection and Estimation for Censored Survival Data with High Dimensional Genomic Covariates
}

\author{
Guorong Chen ${ }^{1}\left[\right.$. S Sijian Wang ${ }^{2} \cdot$ Guannan Sun ${ }^{3} \cdot$ Huanxue Pan ${ }^{1}$
}

Published online: 27 February 2020

c) Springer Nature B.V. 2020

\section{Retraction to: Acta Biotheoretica (2019) 67:225-251 https://doi.org/10.1007/s10441-019-09349-9}

The authors have retracted this article (Chen et al. 2019) because they found a fundamental mistake in the methodology that is not correctable at this time. This mistake is found in the methodology and the derivation of the model with Tukey and Huber's losses. Because of the error, the findings in the article are not reliable. All authors agree to this retraction.

\section{Reference}

Chen G, Wang S, Sun G, Pan H (2019) Robust model selection and estimation for censored survival data with high dimensional genomic covariates. Acta Biotheoretica 67:225-251. https://doi.org/10.1007/ s10441-019-09349-9

Publisher's Note Springer Nature remains neutral with regard to jurisdictional claims in published maps and institutional affiliations.

The original article can be found online at https://doi.org/10.1007/s10441-019-09349-9.

Huanxue Pan

hxpbjfu@163.com

Guorong Chen

cgr1982@163.com

Sijian Wang

sijian.wang@stat.rutgers.edu

Guannan Sun

guannansun2013@163.com

1 Department of Finance, Beijing Forestry University, Beijing, China

2 Department of Statistics and Biostatistics, Rutgers University, New Brunswick, NJ, USA

3 Department of Biostatistics and Programming, Sanofi China, Beijing, China 